\title{
An Interval-based Temporal Logic in a Multivalued Setting
}

\author{
Mathias Bauer
}

July 1992

Deutsches Forschungszentrum für Künstliche Intelligenz $\mathrm{GmbH}$

Postfach 2080

D-6750 Kaiserslautern, FRG

Tel.: (+49 6.31) 205-3211/13

Fax: $(+49631) 205-3210$
Stuhlsatzenhausweg 3

D-6600 Saarbrücken 11, FRG

Tel.: (+49 681) 302-5252

Fax: (+49 681) 302-5341 


\section{Deutsches Forschungszentrum für Künstliche Intelligenz}

The German Research Center for Artificial Intelligence (Deutsches Forschungszentrum für Künstliche Intelligenz, DFKI) with sites in Kaiserslautern and Saarbrücken is a non-profit organization which was founded in 1988. The shareholder companies are Atlas Elektronik, Daimler Benz, Fraunhofer Gesellschaft, GMD, IBM, Insiders, Mannesmann-Kienzle, Philips, SEMA Group Systems, Siemens and Siemens-Nixdorf. Research projects conducted at the DFKI are funded by the German Ministry for Research and Technology, by the shareholder companies, or by other industrial contracts.

The DFKI conducts application-oriented basic research in the field of artificial intelligence and other related subfields of computer science. The overall goal is to construct systems with technical knowledge and common sense which - by using Al methods - implement a problem solution for a selected application area. Currently, there are the following research areas at the DFKI:

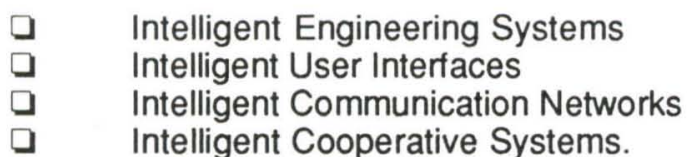

The DFKI strives at making its research results available to the scientific community. There exist many contacts to domestic and foreign research institutions, both in academy and industry. The DFKI hosts technology transfer workshops for shareholders and other interested groups in order to inform about the current state of research.

From its beginning, the DFKI has provided an attractive working environment for $\mathrm{Al}$ researchers from Germany and from all over the world. The goal is to have a staff of about 100 researchers at the end of the building-up phase.

Prof. Dr. Gerhard Barth

Director 


\section{DFKI Documents}

D-91-15

Harold Boley, Philipp Hanschke, Martin Harm,

Knut Hinkelmann, Thomas Labisch, Manfred

Meyer, Jörg Müller, Thomas Oltzen, Michael

Sintek, Werner Stein, Frank Steinle:

mCAD2NC: A Declarative Lathe-Worplanning

Model Transforming CAD-like Geometries into

Abstract NC Programs

100 pages

D-91-16

Jörg Thoben, Franz Schmalhofer, Thomas Reinartz:

Wiederholungs-, Varianten- und Neuplanung bei der

Fertigung rotationssymmetrischer Drehteile

134 Seiten

D-91-17

Andreas Becker:

Analyse der Planungsverfahren der KI im Hinblick auf ihre Eignung für die Abeitsplanung

86 Seiten

\section{D-91-18}

Thomas Reinartz: Definition von Problemklassen im Maschinenbau als eine Begriffsbildungsaufgabe 107 Seiten

D-91-19

Peter Wazinski: Objektlokalisation in graphischen Darstellungen

110 Seiten

D-92-01

Stefan Bussmann: Simulation Environment for

Multi-Agent Worlds - Benutzeranleitung

50 Seiten

\section{D-92-02}

Wolfgang Maaß: Constraint-basierte Plazierung in multimodalen Dokumenten am Beispiel des LayoutManagers in WIP

111 Seiten

D-92-03

Wolfgan Maaß, Thomas Schiffmann, Dudung Soetopo, Winfried Graf: LAYLAB: Ein System zur automatischen Plazierung von Text-BildKombinationen in multimodalen Dokumenten 41 Seiten

\section{D-92-06}

Hans Werner Höper: Systematik zur Beschreibung von Werkstücken in der Terminologie der Featuresprache

392 Seiten

\section{D-92-07}

Susanne Biundo, Franz Schmalhofer (Eds.): Proceedings of the DFKI Workshop on Planning 65 pages
D-92-08

Jochen Heinsohn, Bernhard Hollunder (Eds.):

DFKI Workshop on Taxonomic Reasoning

Proceedings

56 pages

D-92-09

Gernod P. Laufkötter: Implementierungsmöglichkeiten der integrativen Wissensakquisitionsmethode des ARC-TEC-Projektes

86 Seiten

\section{D-92-10}

Jakob Mauss: Ein heuristisch gesteuerter Chart-Parser für attributierte Graph-Grammatiken 87 Seiten

\section{D-92-12}

Otto Kühn, Franz Schmalhofer, Gabriele Schmidt: Integrated Knowledge Acquisition for Lathe

Production Planning: a Picture Gallery (Integrierte Wissensakquisition zur Fertigungsplanung für Drehteile: eine Bildergalerie)

27 pages

\section{D-92-13}

Holger Peine: An Investigation of the Applicability of Terminological Reasoning to ApplicationIndependent Software-Analysis 55 pages

D-92-15

DFKI Wissenschaftlich-Technischer Jahresbericht 1991

130 Seiten

\section{D-92-16}

Judith Engelkamp (Hrsg.)

Verzeichnis von Softwarekomponenten für natürlichsprachliche Systeme 189 Seiten

\section{D-92-17}

Elisabeth André, Robin Cohen, Winfried Graf, Bob Kass, Cécile Paris, Wolfgang Wahlster (Eds.): UM92: Third International Workshop on User Modeling, Proceedings

254 pages

Note: This document is available only for a nominal charge of 25 DM (or 15 US-\$).

\section{D-92-21}

Anne Schauder: Incremental Syntactic Generation of Natural Language with Tree Adjoining Grammars 57 pages 



\title{
An Interval-based Temporal Logic in a Multivalued Setting
}

\author{
Mathias Bauer \\ German Research Center for Artificial Intelligence (DFKI) \\ Stuhlsatzenhausweg 3, \\ W - 6600 Saarbrücken \\ GERMANY \\ bauer@dfki.uni-sb.de
}

\begin{abstract}
We describe the embedding of the semantic notions and modal operators of a first-order temporal logic based on time intervals in a multivalued setting. Truth values will be realized as functions from time intervals to "ordinary" truth values like $t$ and $f$. The main emphasis lies on the realization of the various modal operators contained in the temporal logic as operations on the functional truth values. We show that it is possible to obtain an efficient system sufficient for tasks in the area of diagnostic reasoning.
\end{abstract}




\section{Contents}

1 Introduction 3

2 The Temporal Logic FTL 4

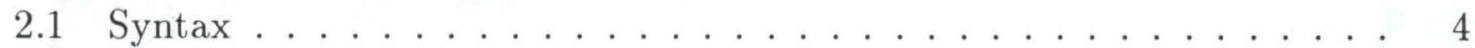

2.2 Semantics of FTL . . . . . . . . . . . . . . . . . . 4

3 Review of MVL and the Embedding of FTL 6

3.1 Mathematical Preliminaries of MVL . . . . . . . . . . . . 7

3.2 Functional Truth Values . . . . . . . . . . . . . . . . . . . 9

3.3 Realization of Modal Operators . . . . . . . . . . . . . . 11

3.4 FTL Modal Operators . . . . . . . . . . . . . . . . 12

3.5 Complexity Considerations . . . . . . . . . . . . . 15

4 Applications $\quad 15$

5 Conclusions $\quad 16$ 


\section{Introduction}

Reasoning about a changing world requires mechanisms going beyond the scope of classical predicate logic. Thus, several temporal logics have been proposed to solve problems in areas like hardware and software verification (e.g., [13]), planning ([2]), reasoning about actions (e.g., [9], [15]), and plan recognition (e.g., [4]).

Among these logics those equipped with operators supporting a compositional approach (e.g., [14], [10]) gained particular importance because they allow some kind of modular reasoning in which formulas can be combined to statements about temporally more complex situations. The operator enabling this kind of reasoning is often referred to as chop $(\mathcal{C})$. The semantics of logics containing it is usually based on the notion of intervals as sequences of states in contrast to logics like tense logic (cf. [5]) based on time points and Allen's temporal logic with intervals that are not built up from single states (cf. [1]).

Unfortunately, decision procedures for propositional temporal logics as described in [14] are non-elementary, i.e., of exponential height in the nesting depth of the chop operator. If the demand for completeness is relaxed, however, it is possible to implement inference systems of clearly smaller complexity for tasks in the field of diagnostic reasoning (e.g., [9], [4]).

The system MVL described in [6] suggests itself as a basis for such an implementation as it provides a proof system to which modal operators like chop can be added (cf. [8]). The crucial idea of MVL (which stands for "Multivalued Logics") is to keep the inference machine and the "bookkeeping" about truth values separated from each other. Thus, exchanging the set of possible truth values while retaining the prover results in a system for a totally different logic.

The aim of this paper is to describe the theoretical foundations of an implementation of FTL, a first-order version of the temporal logic introduced in [14], in the MVL setting and its realization, possible applications, and limitations. The reasons for embedding this logic in MVL are twofold: On the one hand, we will see how MVL generalizes the usual concepts of Kripke-style modal operators and sets of truth values underlying a certain logic (cf. section 3.3). On the other hand, this enables us to give an efficient implementation of a restricted inference machine for FTL (cf. section 3.4).

Sections 2 and 3.1 will introduce syntax and semantics of our temporal logic and the foundations of the MVL system, resp. In sections 3.2 through 3.4, we will describe how to embed our interval-based logic and its modal operators in MVL. As truth values we will use functions from time intervals to "ordinary" values including $t$ and $f$ and demonstrate how complex modal operators can be realized as operations on these functions. We will also address the problem of how to represent the set of intervals and the truth functions so that an effective computation is possible. In section 3.5 , the computational overhead caused by the modal operators is shown to play a minor role concerning the complexity of the whole system. Finally, we will consider limitations and possible applications of the resulting system. 


\section{The Temporal Logic FTL}

The temporal logic FTL ("First-order Temporal Logic") presented in this section essentially corresponds to the extension of the system PTL(U,X,C) as described in [14] to first-order logic.

\subsection{Syntax}

Given a denumerable set $X$ of variables and a signature $S$, the set of formulas of FTL comprises $T, F$, and the usual set of first-order formulas with quantifiers $\forall$ and $\exists$ and the connectives $\neg, \wedge, \vee, \rightarrow$, and $\leftrightarrow$ over $S$ and $X$. Besides, it contains all formulas of the form $O p$ ("next"), $(p \mathcal{U} q)$ ("until"), and $(p \mathcal{C} q)$ ( "chop"). By $\Phi_{0}$ we denote the set of atomic first-order formulas. The versions of next and chop presented here are often referred to as "strong next" and "strong chop".

Before presenting the formal semantics of FTL, we give an intuitive description of the meaning of the modal formulas introduced above: We want to consider a formula $\bigcirc p$ true in an interval $\sigma$ if $p$ is true if we consider the situation one state later, i.e., if $p$ is true in the interval obtained from $\sigma$ by removing its first state.

We say $(p \mathcal{U} q)$ holds in an interval $\sigma$ if $q$ holds sometime within $\sigma$ and $p$ holds all of the time before within $\sigma$.

The chop operator $\mathcal{C}$ provides a possibility to compose two formulas $p$ and $q$ by concatenating the intervals in which they hold. Considered differently, chop allows to split $u p$ an interval $\sigma$ in which $(p \mathcal{C} q)$ holds into two subintervals $\sigma_{1}$ and $\sigma_{2}$ where $p$ and $q$ hold, resp.

\subsection{Semantics of FTL}

Definition 2.1 Any subset of $\Phi_{0}$ is called a state. Let $\Sigma$ be the power set $2^{\Phi_{0}}$ of $\Phi_{0}$. Then the elements of $\mathcal{I}=\Sigma^{+} \cup \Sigma^{\omega}$ are called intervals.

The idea behind this definition is that a state contains just those atomic formulas true at a certain moment in time. Intervals are non-empty sequences of states and thus model the truth or falsity of formulas over time.

We need some operations on intervals:

Definition 2.2 Let $\sigma, \sigma_{1}, \sigma_{2}$ be intervals. Then the length of $\sigma$ is defined by

$$
|\sigma|= \begin{cases}\omega, & \text { if } \sigma \text { is infinite } \\ n, & \text { if } \sigma=\left\langle S_{0}, \ldots, S_{n}\right\rangle\end{cases}
$$

The composition of $\sigma_{1}$ and $\sigma_{2}$ is

$$
\sigma_{1} \oplus \sigma_{2}= \begin{cases}\sigma_{1}, & \text { if }\left|\sigma_{1}\right|=\omega \\ \left\langle S_{0}, \ldots, S_{n}, S_{n+1}, \ldots\right\rangle, & \text { if } \sigma_{1}=\left\langle S_{0}, \ldots, S_{n}\right\rangle \text { and } \sigma_{2}=\left\langle S_{n}, S_{n+1}, \ldots\right\rangle\end{cases}
$$

The $n$th suffix of $\sigma=\left\langle S_{0}, \ldots\right\rangle$ is $\sigma^{(n)}=\left\langle S_{n}, \ldots\right\rangle$. 
Relating FTL to classical modal logics with Kripke-style semantics, we can regard intervals as possible worlds. The accessibility relation between worlds can be described by terminating subintervals defined below:

Definition 2.3 Let $\sigma_{1}, \sigma_{2}$ be intervals. Then we define $R_{t} \subseteq \mathcal{I} \times \mathcal{I}$ by

$$
\sigma_{1} R_{t} \sigma_{2}: \Longleftrightarrow \sigma_{1}=\sigma_{2}^{(1)},
$$

and call the first suffix $\sigma_{1}$ of $\sigma_{2}$ the first terminating subinterval of $\sigma_{2}$.

We denote the transitive closure of $R_{t}$ by $R_{t}^{+}$and the reflexive, transitive closure of $R_{t}$ by $\bar{R}_{t}$.

If $\sigma_{1} \bar{R}_{t} \sigma_{2}$, then there is a unique interval $\sigma^{\prime}$ such that $\sigma_{2}=\sigma^{\prime} \oplus \sigma_{1}$. This interval is the complement of $\sigma_{1}$ in $\sigma_{2}$ and is denoted by $\operatorname{compl}_{\sigma_{2}}\left(\sigma_{1}\right)$.

So, a world $\sigma_{1}$ is accessible from $\sigma$ if $\sigma_{1}$ is a terminating subinterval of $\sigma$. Now we are ready to define the notion of satisfiability.

Definition 2.4 Let $D$ denote the non-empty set over which we interpret the logical variables of $X$. Then $\rho: X \rightarrow D$ is called an assignment. Let $\sigma=\left\langle S_{0}, \ldots\right\rangle \in \mathcal{I}$ be an interval, $x \in X$ a variable, $P \in \Phi_{0}$ an atomic first-order formula, $p, q$ FTL formulas. Then we have

$$
\begin{aligned}
& \sigma \models T \\
& \sigma \not \models F \\
& \sigma \models P \quad \Longleftrightarrow P \in S_{0} \\
& \sigma \models \neg p \quad \Longleftrightarrow \sigma \not \models p \\
& \sigma \models p \wedge q \quad \Longleftrightarrow \sigma \models p \text { and } \sigma \models q \\
& \sigma \models \exists x . p \quad \Longleftrightarrow \text { there is an assignment } \rho \text { such that } \sigma \models p_{\rho(x)}^{x} \\
& \sigma \models O p \quad \Longleftrightarrow \sigma^{(1)} \models p \\
& \sigma \models(p \mathcal{U} q) \Longleftrightarrow \text { there is } \sigma^{\prime \prime}, \sigma^{\prime \prime} \bar{R}_{t} \sigma \text {, such that } \sigma^{\prime \prime} \models q \text { and for all } \sigma^{\prime} \text { : } \\
& \text { if } \sigma^{\prime} \bar{R}_{t} \sigma \text { and } \sigma^{\prime \prime} R_{t}^{+} \sigma^{\prime} \text {, then } \sigma^{\prime} \models p \\
& \sigma \models(p \mathcal{C} q) \Longleftrightarrow \text { there are } \sigma^{\prime}, \sigma^{\prime \prime} \text { such that }\left|\sigma^{\prime}\right|<\omega, \sigma^{\prime} \models p, \sigma^{\prime \prime} \models q \text {, } \\
& \text { and } \sigma=\sigma^{\prime} \oplus \sigma^{\prime \prime}
\end{aligned}
$$

Concerning the other connectives and the universal quantifier, we use the usual recursive definitions.

It should be noted that the truth value of a non-modal formula only depends on the first state of an interval.

On the basis of the operators defined so far, we can derive other modalities useful in temporal reasoning. Examples are

$$
\diamond p: \equiv(T \mathcal{U} p)
$$

and its dual

$$
\square p: \equiv \neg \diamond \neg p .
$$

These operators are called sometimes and always. In "classical" modal logics, they correspond to the modalities possibly and necessarily. Introducing the abbreviation

$$
\text { empty : }: \neg \bigcirc T
$$


to denote the end of an interval, we can derive the so-called weak versions of chop and next:

$$
\begin{array}{ll}
(p \mathcal{C} q): \equiv((p \mathcal{C} q) \vee(p \wedge \square \neg \text { empty })) \\
\odot p \quad: \equiv \text { empty } \vee \bigcirc p .
\end{array}
$$

\section{Review of MVL and the Embedding of FTL}

The MVL system by Ginsberg is an attempt to capture many sorts of reasoning within the field of artificial intelligence in a uniform framework (cf. [6]). The basic idea is to split up inference into two parts: One in which the actual process of reasoning takes place - realized by a theorem prover - and one in which some kind of "bookkeeping" of the results obtained from the inference machine is done.

As an example, one might imagine a system for probabilistic reasoning where the bookkeeping consists of combining the numerical values assigned to the formulas used and pruning formulas whose probability is below a certain threshold. Other examples given by Ginsberg are ATMS and default reasoning systems.

Ginsberg formalizes the bookkeeping part of reasoning by attaching two kinds of labels to each bit of information: one describing the amount of knowledge available about a certain statement and one indicating the degree of certainty about its validity. On the basis of these labels, sets of truth values can be given the internal structure of a so-called bilattice, which is advantageous with regard to several aspects:

1. Modularity. It is possible to develop theorem provers suitable for many different object logics independent of the actual choice of underlying truth values, since the definition of a bilattice forms a unique interface to the bookkeeping part of the inference machine. Selecting a new set of possible truth values yields a reasoner for a totally different logic although the original prover is further used (cf. [7] and [8]).

2. Efficiency. As we will see, it is possible to exploit the additional information represented in the bilattice structure during the inference process to render it more efficient.

3. Modal operators. It is easy to introduce new modal operators into a given logic, as they can essentially be expressed using primitive operations on the elements of a bilattice. Besides forming the basis for efficient implementations, this is also interesting from a theoretical point of view, as this approach generalizes both the classical concept of Kripke-style modal operators and Moore's autoepistemic operator $L$ (cf. [12]). Thus, we are able to introduce modal operators of arity $>1$ (in fact, the FTL operators $\mathcal{U}$ and $\mathcal{C}$ exceed Kripke's approach) and to compare different modal logics within a single uniform framework.

In section 3.1, we will describe the formal basis for the truth values to be chosen and the way in which the closure of a certain set of propositions is computed using this basis. Section 3.2 shows how functions can serve as truth values in this sense and applies these results to FTL. In sections 3.3 and 3.4, we consider the MVL concept of 
modal operators as described in [8] and its application to our temporal logic before we finally give some complexity results in 3.5 .

\subsection{Mathematical Preliminaries of MVL}

The fundamental notion in connection with MVL truth values is that of a bilattice defined below: ${ }^{1}$

Definition $3.1([6])$ A bilattice is a sextuple $(B, \wedge, \vee, \cdot,+, \neg)$ such that

1. $(B, \wedge, \vee)$ and $(B, \cdot,+)$ are both complete lattices.

2. $\neg: B \rightarrow B$ is a mapping with

(a) $\neg^{2}=1$, and

(b) $\neg$ is a lattice homomorphism from $(B, \wedge, \vee)$ to $(B, \vee, \wedge)$ and from $(B, \cdot,+)$ to itself.

If the operations $\wedge, \vee,+$, and $\cdot$ distribute with respect to each other, the bilattice is called distributive. If only $\wedge, \vee$ and $\cdot,+$ each distribute with respect to each other, it is called $t$-distributive and $k$-distributive, resp.

The elements of a bilattice can be considered as truth values if its operations are interpreted in the following way: The two pairs of operations $\wedge, \vee$ and $\cdot,+$ each induce a partial order on the elements of $B$, denoted by $\leq_{t}$ and $\leq_{k}$, resp. If $x$ and $y$ are elements of $B$ with $x \leq_{t} y$, we interpret this by saying that $y$ represents a truth value that is "nearer to truth" than the one represented by $x$. In other words, a formula assigned the truth value $y$ is considered "more true" than one assigned $x$. An example for this ordering is $f \leq_{t} t$. Thus, $\leq_{t}$ represents the degree of certainty about the validity of a certain statement.

If on the other hand $x \leq_{k} y$, we say that $y$ stands for a greater amount of knowledge about a certain fact than $x$. If we allow for some truth value $u$ (unknown), we have $u \leq_{k} f$ and $u \leq_{k} t$, whereas $t$ and $f$ are incomparable with respect to $\leq_{k}$.

Completing the truth values used so far with another element denoted by $\perp$ which stands for "both $t$ and $f$ ", we obtain the smallest non-trivial bilattice $F$ representing the set of truth values used in first-order logic in MVL. Figure 1 shows this bilattice where $\leq_{t}$ increases from left to right and $\leq_{k}$ from bottom to top. The role of the $\neg$ operation is to invert the $\leq_{t}$ order while retaining $\leq_{k}$. We now relate the bilattice operations $\wedge, \vee$, and $\neg$ to the interpretation of the elements of the bilattice as truth values. As the notation indicates, there is a strong similarity between these operations in a bilattice and their syntactical counterparts within logic. Considering the bilattice $F$ for example, we get $t \wedge f=f, t \vee f=t, \neg t=f$ etc., just as expected from traditional logic. ${ }^{2}$

\footnotetext{
${ }^{1}$ The presentation of the mathematical foundations of MVL in this paper has to concentrate on the most essential topics. For an elaborated description, the reader is referred to [6].

${ }^{2} \wedge$ yields the greatest lower bound $g l b_{t}$ of its arguments w.r.t. $\leq_{t}$ and $\vee$ the least upper bound $l u b_{t}$
} 


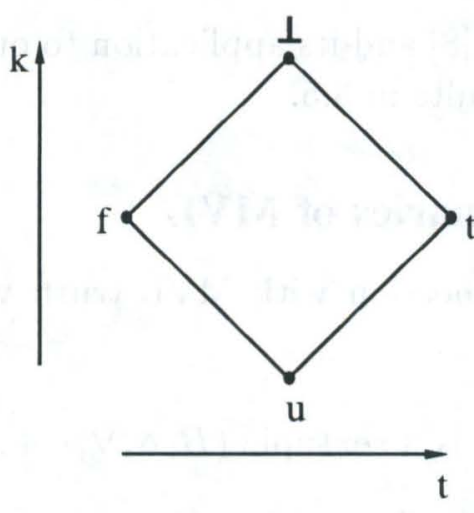

Figure 1: The bilattice $F$ for first-order logic.

Definition 3.2 ([6]) Given some logical language $\mathcal{L}$ and a bilattice $B$, a truth assignment is a mapping $\phi: \mathcal{L} \rightarrow B$.

In MVL, such a mapping corresponds to a declarative database. In conventional logics, inferencing a formula $p$ from a set $S$ of axioms consists in checking if $p$ is a member of the deductive closure of $S$. The multivalued counterpart for this process is to compute the truth value of $p$ in the closure $\operatorname{cl}(\phi)$ of a truth assignment $\phi$.

Definition 3.3 ([6]) A truth assignment $\phi$ is closed if for all $p, q, p_{1}, p_{2}, \ldots \in \mathcal{L}$

1. $\phi\left(\bigwedge_{i} p_{i}\right) \geq_{k} \bigwedge_{i} \phi\left(p_{i}\right)$,

2. $\phi(\neg p)=\neg \phi(p)$, and

3. if $p \models q$, then $\phi(q) \geq_{t} \phi(p)$.

This means that - as already mentioned - the behaviour of the bilattice operation $\neg$ perfectly corresponds to negation in logic (2.), and that a formula $q$ can't be "less true" than a formula $p$ entailing it (3.). The content of clause 1 . becomes clear when considering a formula $p \wedge \neg p$ where both conjuncts are assigned $u$ but their conjunction obviously should be assigned $f$.

According to the "classical" approach to logic where the closure of some set $T$ of sentences is defined to be the intersection of all deductively closed sets containing $T$, we define the closure $c l(\phi)$ of a truth assignment $\phi$ by:

Definition $3.4([6]) c l(\phi)=\prod\left\{\psi \mid \psi \geq_{k} \phi\right.$ and $\psi$ is closed $\}$, where $\phi$ and $\psi$ are compared pointwise.

To give a constructive account of closure, we will restrict ourselves here to the case of so-called canonically grounded bilattices where each element $x$ can be expressed uniquely by a sum $x=g_{t}(x)+g_{f}(x)$, the t-grounding and $f$-grounding of $x$, resp. They correspond to the primitive bits of information $x$ is composed of. ${ }^{3}$ The bilattice $F$ and the one used for FTL are both canonically grounded.

Now let $p$ and $q$ be sentences of our logical language such that $p \models q$ and $\phi(p)=x$. According to definition 3.3 , we have $\operatorname{cl}(\phi)(q) \geq_{t} x$ and thus $c l(\phi)(q) \geq_{k} g_{t}(x)$ (since

\footnotetext{
${ }^{3}$ In the bilattices considered here, $g_{t}(x)=x \vee u, g_{f}(x)=x \wedge u$. E.g., $g_{t}(\perp)=t, g_{f}(\perp)=f$.
} 
$\left.x=g_{t}(x)+g_{f}(x) \geq_{k} g_{t}(x)\right)$. So the contribution of $p$ to $q$ 's truth value is $g_{t}(\phi(p))$. If there are many sentences entailing $q$, this knowledge has to be accumulated by summing over all t-groundings.

For a set $S \subseteq \mathcal{L}$ of sentences we define $\phi(S):=\bigwedge_{p \in S} \phi(p)$. If $p \in \mathcal{L}$, we denote by $\pi_{+}(p)$ and $\pi_{-}(p)$ the sets of all subsets of $\mathcal{L}$ entailing $p$ and $\neg p$, resp.:

$$
\pi_{+}(p)=\{S \mid S \models p\}, \quad \pi_{-}(p)=\{S \mid S \models \neg p\} .
$$

A truth assignment $\phi$ is called $\neg$-closed if $\phi(\neg p)=\neg \phi(p)$ for all $p$. This property can be characterized by the following lemma:

Lemma 3.1 ([6]) A truth assignment $\phi$ is $\neg$-closed iff

$$
\phi(p)=\sum_{q \equiv \neg p} \phi(q)+\sum_{q \equiv \neg \neg p} \neg \phi(q)
$$

for all $p$, where $q \equiv_{\neg} p$ if there exists a nonnegative integer $n$ such that $p=\neg^{2 n} q$ or vice versa.

Now assume we are given a $\neg$-closed truth assignment $\phi .{ }^{4}$ Then we have

Theorem 3.1 ([6]) The closure of $\phi$ is given by

$$
\begin{aligned}
c l(\phi)(p) & =\sum_{S \in \pi_{+}(p)}[\phi(S) \vee u]+\sum_{S \in \pi_{-}(p)}[\neg \phi(S) \wedge u] \\
& =\sum_{S \in \pi_{+}(p)} g_{t}[\phi(S)]+\sum_{S \in \pi_{-}(p)} \neg g_{t}[\phi(S)]
\end{aligned}
$$

This result implies a method to effectively compute the closure by steadily pruning formulas from the search space whose truth value is $<_{k}$ than the truth value already accumulated during the previous steps of the proof, since they can't make a real contribution to $\operatorname{cl}(\phi)(p)$. Furthermore, the summation over $\pi_{-}(p)$ may be left out if we are only interested in the truth of $p$, i.e., if we want to show $\operatorname{cl}(\phi)(p) \geq_{k} t$.

\subsection{Functional Truth Values}

For some applications it is not sufficient to use some kind of "atomic" truth values like $t$ and $f$. Instead, it might be convenient to employ mappings from a given set to some bilattice as truth values. Ginsberg describes this for a simple temporal logic with truth values $g: \mathbb{N} \rightarrow F$ from the set of time points to the classical truth values (cf. [8], [9]). After describing the principal concept of functional truth values, we will see in this section how this technique can be applied to FTL.

Given a set $S$ and a bilattice $B$, the set $B^{S}$ of functions from $S$ to $B$ obviously inherits the bilattice property from $B$ if the operations $\wedge, \vee, \cdot,+, \neg$ are computed pointwise.

Taking $S$ to be the set of time intervals represented by $I N^{2}$, and $B$ to be the firstorder bilattice $F$, we obtain the new bilattice of truth values of FTL, denoted by $B_{I}$.

\footnotetext{
${ }^{4}$ If $\phi$ doesn't meet this property, we compute its $\neg$ closure according to lemma 3.1 .
} 
Intervals are represented as pairs of natural numbers $i=(a, l)$, where $a$ is the first state and $l$ is the number of states of $i$. Thus, the elements of $B_{I}$ are total functions over $\mathbb{N}^{2}$.

Some practical problems arise with this approach: How to represent the infinitely large set $\mathbb{N}^{2}$ and the functions $g: \mathbb{N}^{2} \rightarrow F$ ?

We begin with the second question and assume the general case of a bilattice $B^{S}$. If we can put some order on the set $S$, it is possible to make the representation of the functions $g$ more compact by only listing those points of $S$ explicitly where the value of $g$ changes and assuming $g$ to be constant between two such so-called exception points. As in general there will be no natural total order available for $S$, we organize $S$ as a directed acyclic graph (DAG) with an induced partial order $\preceq$ :

$$
s_{1} \preceq s_{2}: \Longleftrightarrow \text { there is a path from } s_{1} \text { to } s_{2} \text { in } S \text {. }
$$

To effectively represent this DAG, it is sufficient to have its root and a function computing the common successors of a given pair of points.

The required structure for the interval DAG $D_{I}$ can be extracted from definition 2.4. As our time begins with state 0 , we define the root of $D_{I}$ to be $(0,1)$, i.e., the interval consisting only of state 0 . Examining those intervals sharing some common properties yields four classes:

1. Intervals of the form $(a, 1)$ consisting of a single state. They correspond to the embedding of time points into intervals. Within $D_{I}$, they yield a path $(0,1) \rightarrow$ $(1,1) \rightarrow(2,1) \rightarrow \ldots$ called the $\alpha$-branch of $D_{I}$.

2. Intervals beginning with the same state, as a non-modal formula is valid in $(a, l)$ iff it is in $\left(a, l^{\prime}\right)$ for any $l^{\prime}>0$. For each $a \in \mathbb{N}$, these form a path $(a, 1) \rightarrow$ $(a, 2) \rightarrow(a, 3) \rightarrow \ldots$, the $\beta_{a}$-branch of $D_{I}$.

3. Intervals ending in the same state. They all are terminating subintervals of a common greater interval and form pathes $(0, l) \rightarrow(1, l-1) \rightarrow \ldots \rightarrow(l-1,1)$. A sequence of this form is called the $\gamma_{l}$-branch of $D_{I}$ as for all $\left(a^{\prime}, l^{\prime}\right) \in \gamma_{l}$ : $a^{\prime}+l^{\prime}=l$. By $\gamma_{a, l}$ we denote that part of $\gamma_{a+l}$ beginning with $(a, l)$.

4. "Empty" intervals $(a, 0)$. They are only mentioned for completeness as any formula is considered unknown in such an interval. In the following, we won't regard this kind of intervals anymore.

Figure 2 shows a part of the resulting graph $D_{I}$.

This structure of $D_{I}$ allows to pass truth information along the edges to represent all kinds of relations between intervals described in definition 2.4. We now demonstrate how the concepts developed so far can be applied.

Example 3.1 Let $p$ be a first-order formula true in state 2 and unknown everywhere else. According to definition $2.4, p$ is true in all intervals beginning with state 2 . This can be expressed by assigning $p$ a truth function $g_{1}: N^{2} \rightarrow F$ with

$$
g_{1}((a, l))= \begin{cases}t, & a=2, l \geq 1 \\ u, & \text { otherwise }\end{cases}
$$




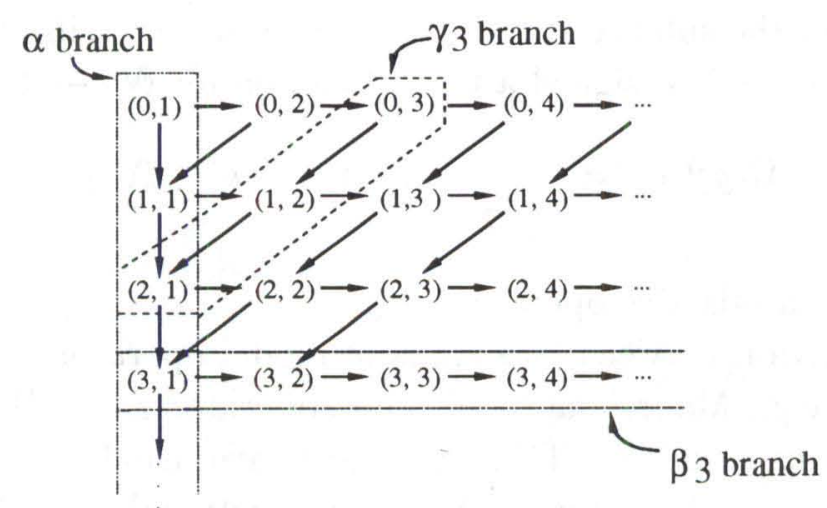

Figure 2: The DAG $D_{I}$ of intervals.

Listing $g_{1}$ 's exception points and their respective truth values yields $\langle(0,1)-u,(2,1)-$ $t,(3,1)-u\rangle$. In this case, we use inheritance along the $\beta_{2}$ branch of $D_{I}$ and the fact that the interval $(2,1)$ of the $\alpha$ branch corresponds to state 2 .

If we want to express that an FTL formula $q$ is true in all states of an interval, e.g., $(5,3)$, we use a truth function $g_{2}$ with

$$
g_{2}(w)= \begin{cases}t, & w \in\{(5,3),(6,2),(7,1)\} \\ u, & \text { otherwise }\end{cases}
$$

The exception point list of $g_{2}$ is $\langle(0,1)-u,(5,3)-t,(5,4)-u\rangle$, where $t$ is passed along the $\gamma_{5,3}$ branch of $D_{I}$.

\subsection{Realization of Modal Operators}

As already mentioned in section 3.1 , the bilattice operations like " $\wedge$ " play two distinct roles: Besides being a function on elements of a bilattice, they occur as binary operators in our logical language. In [8], Ginsberg generalizes this view to arbitrary operations:

"...bilattice operations can be viewed in general as establishing semantic meanings for their syntactic counterparts. These syntactic counterparts are generally referred to as modal operators."

Definition 3.5 ([8]) Given a bilattice $B$, any $n$-ary function $g: B^{n} \rightarrow B$ is a modal operator.

Usually, modal operators are given a semantics using Kripke's approach of possible worlds (cf.[11]). Given an accessibility relation $r$ among worlds and a modal operator $\Omega$, where $\Omega p$ is intended to be valid in a world $w$ if $p$ holds in all worlds $w^{\prime}$ accessible from $w$, we can define the semantics of this operator by conceptually introducing a function $\Gamma$ that takes a formula $p$ and a world $w$ and returns the truth value of $p$ in $w .^{5}$ Then we have

$$
\Gamma(\Omega p, w)=\bigwedge_{\substack{w^{\prime} \\ r\left(w, w^{\prime}\right)}} \Gamma\left(p, w^{\prime}\right) .
$$

\footnotetext{
${ }^{5} \Omega$ corresponds to the classical "necessity" operator.
} 
How can this be related to the results of the previous section? Taking time intervals as possible worlds and the subinterval relationship $\bar{R}_{t}$ as accessibility relation, ${ }^{6}$ we get for a formula $\Omega p$, where $p$ is assigned a truth function $g: N^{2} \rightarrow F$ :

$$
\Omega(g)(w)=\bigwedge_{w^{\prime} \bar{R}_{t} w} g\left(w^{\prime}\right) \quad\left(w \in N^{2}\right)
$$

In this equation, $\Omega$ is a bilattice operator.

There exists a distinction between two kinds of modal operators: The so-called nondeductive operators, e.g., Moore's autoepistemic operator $L$ (cf. [12]), do not respect the bilattice operations - and + . These operators are usually not given a possibleworlds semantics, but are characterized by some functional relationship between the truth values of their "input" formulas and their results. The class of deductive modal operators is characterized by the classical Kripke semantics and comprises operators like those of necessity and possibility. When computing the closure $\operatorname{cl}(\phi)$ of a truth assignment $\phi$, the following inequality holds for deductive operators $\Omega$ :

$$
\operatorname{cl}(\phi)\left(\Omega\left(p_{1}, \ldots, p_{n}\right)\right) \geq_{k} \Omega\left(\operatorname{cl}(\phi)\left(p_{1}\right), \ldots, \operatorname{cl}(\phi)\left(p_{n}\right)\right),
$$

while there is a strict equality for the non-deductive ones.

Proposition 3.1 ([8]) Any modal operator on a distributive bilattice $B$ that can be written in terms of $\wedge, \neg,+$, constant functions and projections is deductive.

Projections are functions $\pi_{w, w^{\prime}}$ indexed by two possible worlds $w, w^{\prime}$ that - when supplied with a truth function $g$ and a world $w^{\prime \prime}$ - return the value of $g$ at $w^{\prime}$ if $w=w^{\prime \prime}$ and $u$ otherwise; i.e., such a projection exactly represents the property of accessibility of $w^{\prime}$ from $w$.

\subsection{FTL Modal Operators}

In the following, we will describe how the basic modal operators of FTL introduced in section 2 can be expressed as bilattice operations on $B_{I}$.

Let $g, g_{1}, g_{2} \in B_{I}$ be truth functions $\mathbb{N}^{2} \rightarrow F$, and $w, w^{\prime}, w^{\prime \prime} \in \mathbb{N}^{2}$ pairs of natural numbers representing intervals.

Then we can express the next operator $\bigcirc$ by

$$
\operatorname{next}(g)(w)=g\left(w^{\prime}\right)
$$

where $w^{\prime}$ is uniquely determined by $w^{\prime} R_{t} w$. This corresponds to the intuitive semantics of next: To see if $O p$ is valid in an interval $w$, check $p$ 's truth value in its first terminating subinterval $w^{\prime}$. Obviously, the result of next is itself an element of $B_{I}$.

With the other basic operators, things become slightly more complicated. Consider the operator until. Translating its semantics given in definition 2.4 into the MVL formalism yields the following: Given two truth functions $g_{1}, g_{2} \in B_{I}$, we can determine

\footnotetext{
${ }^{6}$ I.E., $w^{\prime}$ is accessible from $w$ iff $w^{\prime} \bar{R}_{t} w$.
} 
the value of $\operatorname{until}\left(g_{1}, g_{2}\right)$ at $w$ by checking if there is some terminating subinterval $w^{\prime \prime}$ such that $g_{2}\left(w^{\prime \prime}\right)=t$ and for all subintervals $w^{\prime}$ of $w$ before $w^{\prime \prime}: g_{1}\left(w^{\prime}\right)=t$. We get

$$
\operatorname{until}\left(g_{1}, g_{2}\right)(w)=\bigvee_{w^{\prime \prime} \bar{R}_{t} w}\left[\bigwedge_{\substack{w^{\prime} \bar{R}_{t} w \\ w^{\prime \prime} R_{t}^{+} w^{\prime}}} g_{1}\left(w^{\prime}\right) \wedge g_{2}\left(w^{\prime \prime}\right)\right] .
$$

Remark: In (2), $l u b_{t}$ and $g l b_{t}$ are guaranteed to exist even in the infinite case because - according to definition $3.1-\left(B_{I}, \wedge, \vee\right)$ is a complete lattice. In contrast to traditional logic, we can therefore express existential and universal propositions about worlds by disjunctions and conjunctions, resp.

For the chop operator, the translation is similar: Check if there is a terminating subinterval $w^{\prime}$ of $w$ such that $g_{2}\left(w^{\prime}\right)=t$ and for the complement $w^{\prime \prime}$ of $w^{\prime}$ in $w$ : $g_{1}\left(w^{\prime \prime}\right)=t$. Again, we get a disjunction

$$
\operatorname{chop}\left(g_{1}, g_{2}\right)(w)=\bigvee_{w^{\prime} \bar{R}_{t} w}\left[g_{1}\left(\operatorname{compl}_{w}\left(w^{\prime}\right)\right) \wedge g_{2}\left(w^{\prime}\right)\right]
$$

Here, the above remark is also valid yielding the correctness of (3). As a consequence of (1), (2), (3), and proposition 3.1, we get the following result:

Corollary 3.1 The modal operators next $(\bigcirc)$, until $(\mathcal{U})$, and chop $(\mathcal{C})$ are deductive.

From the equations listed above, an actual implementation of these operators can be easily derived. For this, notice that

1. $\left(a^{\prime}, l^{\prime}\right) R_{t}(a, l) \Longleftrightarrow l>1, a^{\prime}=a+1$, and $l^{\prime}=l-1$.

2. $\left(a^{\prime}, l^{\prime}\right) \bar{R}_{t}(a, l) \Longleftrightarrow a+l=a^{\prime}+l^{\prime}, l^{\prime}>0$, and $a^{\prime} \geq a$.

3. $\left(a^{\prime}, l^{\prime}\right) R_{t}^{+}(a, l) \Longleftrightarrow a+l=a^{\prime}+l^{\prime}, l^{\prime}>0$, and $a^{\prime}>a$.

4. $\operatorname{compl}_{(a, l)}((a+i, l-i))=(a, i+1)$.

So we get

$$
\operatorname{next}(g)((a, l))=g((a+1, l-1))
$$

and

$$
\operatorname{until}\left(g_{1}, g_{2}\right)((a, l))=\bigvee_{i=0}^{l-1}\left[\bigwedge_{j=0}^{i-1} g_{1}((a+j, l-j)) \wedge g_{2}((a+i, l-i))\right]
$$

The implementation of chop is done by

$$
\operatorname{chop}\left(g_{1}, g_{2}\right)((a, l))=\bigvee_{i=0}^{l-1}\left[g_{1}((a, i+1)) \wedge g_{2}((a+i, l-i))\right] .
$$

To complete this section, we will consider the derived modal operators always ( $\square$ ) and sometimes $(\diamond)$. As mentioned in section 2 , sometimes can be obtained by

$$
\diamond p: \equiv(T \mathcal{U} p)
$$


Denoting the truth functions assigned to $T$ and $p$ by $g_{T}$ and $g_{p}$, resp. (i.e., $\forall w \in \mathbb{N}^{2}$ : $g_{T}(w)=t$, and inserting them into $(2)$, we immediately get

$$
\begin{aligned}
\text { sometimes }\left(g_{p}\right)(w) & =\operatorname{until}\left(g_{T}, g_{p}\right)(w) \\
& \left.=\bigvee_{w^{\prime \prime} \bar{R}_{t} w}\left[\bigwedge_{\substack{w^{\prime} \bar{R}_{t} w \\
w^{\prime \prime} R_{t}^{+} w^{\prime}}} t \wedge g_{p}\left(w^{\prime \prime}\right)\right] \quad \text { (cf. def. of } g_{T}\right) \\
& =\bigvee_{w^{\prime \prime} \bar{R}_{t} w} g_{p}\left(w^{\prime \prime}\right)
\end{aligned}
$$

Taking advantage of the duality of sometimes and always, it is possible to derive its realization by

$$
\begin{aligned}
\operatorname{always}(g)(w) & =\neg \text { sometimes }(\neg g)(w) \\
& =\neg \bigvee_{w^{\prime} \bar{R}_{t} w} \neg g\left(w^{\prime}\right) \\
& =\bigwedge_{w^{\prime} \bar{R}_{t} w} g\left(w^{\prime}\right)
\end{aligned}
$$

In general, formulas like (2) and (3) cannot be effectively computed as they may contain infinitely large disjunctions and conjunctions. The representation of the set of intervals in a DAG and of the truth functions by only listing their exception points, however, admits these computations for many cases.

Example 3.2 In example 3.1, we assigned a truth function $g_{2}$ to a formula $q$ with the intention to express that $q$ is true during the whole interval (5,3). Applying always to this function yields always $\left(g_{2}\right)=g_{2}$, i.e., $g_{2}$ in fact formalized what we intended.

Consider another formula $r$ with truth function $g_{3}$ where

$$
g_{3}(w)= \begin{cases}t, & w \in\{(7,2),(8,1)\} \\ u, & \text { otherwise }\end{cases}
$$

i.e., $r$ is true in the whole interval $(7,2)$. Then the truth value of $(q \mathcal{C} r)$ is a function $\operatorname{chop}\left(g_{2}, g_{3}\right)=g_{4}$ with

$$
g_{4}(w)= \begin{cases}t, & w \in\{(5,4),(6,3),(7,2)\} \\ u, & \text { otherwise }\end{cases}
$$

that is represented using the exception points $\langle(0,1)-u,(5,4)-t,(5,5)-u\rangle$. Applying next to $g_{3}$, i.e., computing the truth value of $\mathrm{O}$, yields $n \operatorname{ext}\left(g_{3}\right)=g_{5}$ with

$$
g_{5}(w)= \begin{cases}t, & w \in\{(6,3),(7,2)\} \\ u, & \text { otherwise }\end{cases}
$$

We have a slightly different view on modal operators than Ginsberg has. According to his understanding of modal operators, next should modify the truth value of $r$ by pushing it one step into the future, whereas the above result is the truth function obtained from the query "In which intervals is Or true?" 


\subsection{Complexity Considerations}

For the case of chop, we will describe an algorithm for (6) exploiting the sparsity of exception points of truth functions and compute its complexity.

Let $g_{1}, g_{2} \in B_{I}, \operatorname{exc}\left(g_{1}\right), \operatorname{exc}\left(g_{2}\right)$ their respective sets of exception points with $\left|\operatorname{exc}\left(g_{1}\right)\right|=n_{1}$ and $\left|\operatorname{exc}\left(g_{2}\right)\right|=n_{2}$. As indicated in equation (6), to compute the value of $\operatorname{chop}\left(g_{1}, g_{2}\right)$ at $(a, l)$, we have to consider each possible splitting of $(a, l)$ into two subintervals $\left(a_{1}, l_{1}\right)$ and $\left(a_{2}, l_{2}\right)$ such that $(a, l)=\left(a_{1}, l_{1}\right) \oplus\left(a_{2}, l_{2}\right)$ and combine the respective values of $g_{1}$ and $g_{2}$ using " $\wedge$ ". All these intermediate results are put together in one disjunction yielding the final value at $(a, l)$. In a first step, we have to complete both sets in the following way:

1. Each $\left(a_{2}, l_{2}\right) \in \operatorname{exc}\left(g_{2}\right)$ can be combined with $\left(a_{1}, l_{1}\right) \in \operatorname{exc}\left(g_{1}\right)$ iff $a_{1}+l_{1}-1=$ $a_{2}$, i.e., iff $\left(a_{1}, l_{1}\right) \in \gamma_{a_{2}+1}$. So, we need all points on $\gamma_{a_{2}+1}$ where $g_{1}$ changes its value. Apart from the points of $\gamma_{a_{2}+1} \cap \operatorname{exc}\left(g_{1}\right)$, these are all those elements $\left(a_{1}, l_{1}\right)$ of $\operatorname{exc}\left(g_{1}\right)$ passing their truth values into $\gamma_{a_{2}+1}$ by inheritance. We have to collect their projections $\left(a_{1}, a_{2}-a_{1}+1\right)$ onto $\gamma_{a_{2}+1}$. In the worst case, we therefore have to consider each member of $\operatorname{exc}\left(g_{1}\right)$ for each of $\operatorname{exc}\left(g_{2}\right)$ and obtain a complexity of $O\left(n_{1} \cdot n_{2}\right)$.

2. In the other direction, any $\left(a_{1}, l_{1}\right) \in \operatorname{exc}\left(g_{1}\right)$ is combinable with any $\left(a_{2}, l_{2}\right) \in$ $\operatorname{exc}\left(g_{2}\right)$ where $a_{1}+l_{1}-1=a_{2}$, i.e., with all members of $\beta_{a_{2}}$. Just as in 1 ., we have to compute the projections of all points $\left(a_{2}^{\prime}, l_{2}^{\prime}\right)$ of $\operatorname{exc}\left(g_{2}\right)$ influencing the truth values along $\beta_{a_{2}}$. These are the points $\left(a_{2}, a_{2}^{\prime}+l_{2}^{\prime}-a_{2}\right)$. The complexity of this step is again $O\left(n_{1} \cdot n_{2}\right)$.

3. Combining the points obtained from the two previous steps according to (6) yields the same complexity again.

So, the computational overhead caused by chop is merely $O\left(n_{1} \cdot n_{2}\right)$. For until, the process is similar and takes the same time, whereas next can be implemented to consume linear time.

\section{Applications}

One possible application for a system as the one described is in the field of plan recognition. Assume we are given some observed actions $a_{1}\left(t_{1}\right)$ and $a_{2}\left(t_{2}\right)$ with their actual parameters $t_{i}$ and exact temporal information about their occurrences and some plan hypotheses $P_{1}$ and $P_{2}$ written as FTL formulas, where

$$
\begin{aligned}
& \forall x_{1}, x_{2}, x_{3} \cdot P_{1}\left(x_{1}, x_{2}, x_{3}\right) \equiv\left(a_{1}\left(x_{1}\right) \wedge \diamond\left(a_{2}\left(x_{2}\right) \mathcal{C} a_{3}\left(x_{3}\right)\right)\right) \\
& \forall x_{1}, x_{2}, x_{3} . P_{2}\left(x_{1}, x_{2}, x_{3}\right) \equiv\left(a_{1}\left(x_{1}\right) \wedge \bigcirc a_{2}\left(x_{2}\right) \wedge \bigcirc \bigcirc a_{3}\left(x_{3}\right)\right)
\end{aligned}
$$

Using ordinary deduction, we can infer which of $P_{1}$ and $P_{2}$ is not a valid hypothesis for an explanation of the observed action sequence. Assume $a_{1}\left(t_{1}\right)$ is observed in state 5 , i.e., in the interval $(5,1)$, and $a_{2}\left(t_{2}\right)$ in state 10 . Trying to derive $\neg P_{1}$ from this database yields a truth function $g_{P_{1}}$ with value $t$ everywhere except for all intervals 
$(5, l)$ of $\beta_{5}$ with $l \geq 6$, where $g_{1}$ yields $u$. The reason for this result is the fact that any interval in which $P_{1}$ could hold has to begin with state 5 and include at least state 10 where $a_{2}\left(t_{2}\right)$ takes place. The derivation of $\neg P_{2}$, however, yields a constant truth function $g_{P_{2}}$ with value $t$. The result of these inferences is that $P_{1}$ is consistent with the observations and thus a valid hypothesis for the observed action sequence in contrast to $P_{2}$ that is not.

If later on action $a_{3}\left(t_{3}\right)$ is observed - e.g., at state 12 - we can even derive $P_{1}$ with a truth function $g_{P_{1}}^{\prime}$ that yields $t$ for all $(5, l)$ with $l \geq 8$ as now each interval satisfying $P_{1}$ starts at state 5 and includes at least state 12 .

In [4], a similar approach to plan recognition with a temporal modal logic is described.

\section{Conclusions}

We introduced a modal temporal logic FTL based on work described in [14] and the basic concepts of Ginsberg's MVL presented in [6]. The main emphasis lay on the translation of FTL into the MVL formalism, where the choice of functions $\mathbb{N}^{2} \rightarrow F$ as truth values - as counterparts for its interval-based semantics - and the implementation of FTL's modal operators as functions over. these truth values played a central role. We finally showed that it is even possible to give efficient implementations for these concepts by exploiting some constraints on the structure of truth values.

As expected, this efficiency is not for free. For example, truth functions changing their value infinitely often (e.g., from state to state) can't be represented using the methods described.

Another - perhaps even more serious - drawback lies in the limitation of possible inferences caused by MVL. To reason about a formula $\Omega p$ containing a modal operator $\Omega, p$ is required to have a concrete truth value that can serve as input for the function $\Omega$. Axiom schemata like $\bigcirc A \rightarrow \diamond A$ are conceptually not supported. Thus, the applicability of inference systems based on MVL is limited to cases of diagnostic reasoning, where a set of observations with their actual truth values is given. In such a situation, tasks like temporal projection are also solvable by using modal operators that "push" certain truth values into the future. Examples are reasoning about actions (e.g., Ginsberg's treatment of the Yale Shooting Problem (cf. [9] and [3])), plan recognition as described in [4], and all kinds of fault diagnosis. For such tasks, a more powerful - and less efficient - prover is generally not needed, but can be replaced by an inference system as the one described above.

\section{Acknowledgements}

I'd like to thank Gaby Merziger and Dietmar Dengler for fruitful discussions and Jana köhler for her comments on an earlier version of this paper.

\section{References}

[1] J. Allen. Towards a General Theory of Action and Time. Artif. Intell., 23:123154,1984 .

[2] J. Allen. Planning as Temporal Reasoning. In Proc. of the 2nd International Conference on Principles of Knowledge Representation and Reasoning, 1991. 
[3] A. Baker and M. Ginsberg. Temporal Projection and Explanation. In Proc. of the 11th International Joint Conference on AI, 1989.

[4] M. Bauer, S. Biundo, D. Dengler, M. Hecking, J. Köhler, and G. Merziger. Integrated Plan Generation and Recognition - a Logic-based Approach. In W. Brauer, editor, 4. Internationaler GI-Kongreß Wissensbasierte Systeme. Springer, 1991.

[5] J. Burgess. Basic Tense Logic. In D. Gabbay and F. Guenthner, editors, Handbook of Philosophical Logic, Vol. II, pages 89-133, 1984.

[6] M. Ginsberg. Multivalued Logics: A Uniform Approach to Inference in AI. Computational Intelligence, 4:265-316, 1988.

[7] M. Ginsberg. A Circumscriptive Theorem Prover. Artif. Intell., 39:209-230, 1989.

[8] M. Ginsberg. Bilattices and Modal Operators. J. of Logic and Computation, 1, 1990 .

[9] M. Ginsberg. Computational Considerations in Reasoning about Action. In Proc. of the 2nd International Conference on Principles of Knowledge Representation and Reasoning, 1991.

[10] R. Hale. Temporal Logic Programming. In A. Galton, editor, Temporal Logics and Their Applications, pages 91-119. Academic Press, 1987.

[11] S. Kripke. Semantical Considerations on Modal Logic. Acta Philosophica Fennica, 16:83-94, 1963.

[12] R. Moore. Semantical Considerations on Nonmonotonic Logic. Artif. Intell., 25:75-94, 1985.

[13] B. Moszkowski. A Temporal Logic for Multi-level Reasoning about Hardware. Computer, 18:10-19, 1985.

[14] R. Rosner and A. Pnueli. A Choppy Logic. In Proc. of the Symposium on Logic in Computer Science, pages 306-313, June 1986.

[15] Y. Shoham. Time for Action: On the Relation between Time, Knowledge and Action. In Proc. of the 11th International Joint Conference on AI, pages 954-960, 1989 . 


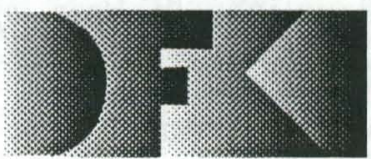

Deutsches

Forschungszentrum

für Künstliche

Intelligenz G mbH
DFKI

-Bibliothek-

PF 2080

D-6750 Kaiserslautern

FRG

\section{DFKI Publikationen}

Die folgenden DFKI Veröffentlichungen sowie die aktuelle Liste von allen bisher erschienenen Publikationen können von der oben angegebenen Adresse bezogen werden.

Die Berichte werden, wenn nicht anders gekennzeichnet, kostenlos abgegeben.

\section{DFKI Research Reports}

R R - 91- 17

Andreas Dengel, Nelson M. Mattos:

The Use of Abstraction Concepts for Representing and Structuring Documents

17 pages

R R -91-18

John Nerbonne, Klaus Netter, Abdel Kader Diagne, Ludwig Dickmann, Judith Klein:

A Diagnostic Tool for German Syntax

20 pages

RR -91-19

Munindar P. Singh: On the Commitments and Precommitments of Limited Agents

15 pages

R R-91-20

Christoph Klauck, Ansgar Bernardi, Ralf Legleitner FEAT-Rep: Representing Features in CAD/CAM 48 pages

\section{RR-91-21}

Klaus Netter: Clause Union and Verb Raising Phenomena in German

38 pages

RR-91-22

Andreas Dengel: Self-Adapting Structuring and Representation of Space

27 pages

RR-91-23

Michael Richter, Ansgar Bernardi, Christoph Klauck, Ralf Legleitner: Akquisition und Repräsentation von technischem Wissen für Planungsaufgaben im Bereich der Fertigungstechnik 24 Seiten

\section{DFKI Publications}

The following DFKI publications or the list of all publisched papers so far can be ordered from the above address.

The reports are distributed free of charge except if otherwise indicated.

\section{R R -91-24}

Jochen Heinsohn: A Hybrid Approach for Modeling Uncertainty in Terminological Logics 22 pages

R R -91-25

Karin Harbusch, Wolfgang Finkler, Anne Schauder: Incremental Syntax Generation with Tree Adjoining Grammars

16 pages

RR -91-26

M. Bauer, S. Biundo, D. Dengler, M. Hecking, J. Koehler, G. Merziger:

Integrated Plan Generation and Recognition - A Logic-Based Approach -

17 pages

R R -91-27

A. Bernardi, H. Boley, Ph. Hanschke,

K. Hinkelmann, Ch. Klauck, O. Kühn, R. Legleitner, M. Meyer, M. M. Richter, F. Schmalhofer, G. Schmidt, W. Sommer: ARC-TEC: Acquisition, Representation and Compilation of Technical Knowledge 18 pages

R R-91-28

Rolf Backofen, Harald Trost, Hans Uszkoreit: Linking Typed Feature Formalisms and Terminological Knowledge Representation Languages in Natural Language Front-Ends 11 pages

R R-91-29

Hans Uszkoreit: Strategies for Adding Control Information to Declarative Grammars

17 pages 
RR -91-30

Dan Flickinger, John Nerbonne:

Inheritance and Complementation: A Case Study of Easy Adjectives and Related Nouns

39 pages

\section{RR-91-31}

H.-U. Krieger, J. Nerbonne:

Feature-Based Inheritance Networks for

Computational Lexicons

11 pages

\section{RR-91-32}

Rolf Backofen, Lutz Euler, Günther Görz:

Towards the Integration of Functions, Relations and Types in an AI Programming Language 14 pages

\section{RR-91-33}

Franz Baader, Klaus Schulz:

Unification in the Union of Disjoint Equational

Theories: Combining Decision Procedures

33 pages

R R-91-34

Bernhard Nebel, Christer Bäckström:

On the Computational Complexity of Temporal

Projection and some related Problems

35 pages

\section{RR-91-35}

Winfried Graf, Wolfgang Maaß: Constraint-basierte Verarbeitung graphischen Wissens

14 Seiten

\section{RR-92-01}

Werner Nutt: Unification in Monoidal Theories is Solving Linear Equations over Semirings 57 pages

R R -92-02

Andreas Dengel, Rainer Bleisinger, Rainer Hoch, Frank Hönes, Frank Fein, Michael Malburg:

$\Pi_{\mathrm{ODA}}$ : The Paper Interface to ODA

53 pages

R R - $92-03$

Harold Boley:

Extended Logic-plus-Functional Programming 28 pages

\section{R R-92-04}

John Nerbonne: Feature-Based Lexicons: An Example and a Comparison to DATR 15 pages

\section{R R-92-05}

Ansgar Bernardi, Christoph Klauck, Ralf Legleitner, Michael Schulte, Rainer Stark: Feature based Integration of CAD and CAPP 19 pages
RR -92-06

Achim Schupetea: Main Topics of DAI: A Review 38 pages

RR -92-07

Michael Beetz:

Decision-theoretic Transformational Planning 22 pages

R R-92-08

Gabriele Merziger: Approaches to Abductive Reasoning - An Overview -

46 pages

\section{R R-92-09}

Winfried Graf, Markus A. Thies:

Perspektiven zur Kombination von automatischem Animationsdesign und planbasierter Hilfe 15 Seiten

R R -92-10

M. Bauer: An Interval-based Temporal Logic in a Multivalued Setting

17 pages

\section{RR -92-11}

Susane Biundo, Dietmar Dengler, Jana Koehler: Deductive Planning and Plan Reuse in a Command Language Environment

13 pages

\section{R R -92-13}

Markus A. Thies, Frank Berger:

Planbasierte graphische Hilfe in objektorientierten Benutzungsoberflächen

13 Seiten

R R - 92 - 14

Intelligent User Support in Graphical User Interfaces:

1. InCome: A System to Navigate through Interactions and Plans

Thomas Fehrle, Markus A. Thies

2. Plan-Based Graphical Help in ObjectOriented User Interfaces 22 pages Markus A. Thies, Frank Berger

\section{RR-92-15}

Winfried Graf: Constraint-Based Graphical Layout of Multimodal Presentations

23 pages

R R-92-16

Jochen Heinsohn, Daniel Kudenko, Berhard Nebel, Hans-Jürgen Profitlich: An Empirical Analysis of Terminological Representation Systems 38 pages 
RR-92-17

Hassan Aït-Kaci, Andreas Podelski, Gert Smolka: A Feature-based Constraint System for Logic Programming with Entailment

23 pages

RR-92-18

John Nerbonne: Constraint-Based Semantics

21 pages

RR-92-19

Ralf Legleitner, Ansgar Bernardi, Christoph Klauck PIM: Planning In Manufacturing using Skeletal Plans and Features

17 pages

RR-92-20

John Nerbonne: Representing Grammar, Meaning and Knowledge

18 pages

RR-92-21

Jörg-Peter Mohren, Jürgen Müller

Representing Spatial Relations (Part II) -The

Geometrical Approach

25 pages

RR-92-22

Jörg Würtz: Unifying Cycles

24 pages

RR-92-24

Gabriele Schmidt: Knowledge Acquisition from

Text in a Complex Domain

20 pages

RR-92-25

Franz Schmalhofer, Ralf Bergmann, Otto Kühn,

Gabriele Schmidt: Using integrated knowledge acquisition to prepare sophisticated expert plans for their re-use in novel situations

12 pages

RR-92-26

Franz Schmalhofer, Thomas Reinartz,

Bidjan Tschaitschian: Intelligent documentation as a catalyst for developing cooperative knowledge-based systems

16 pages

R R-92-27

Franz Schmalhofer, Jörg Thoben: The model-based construction of a case-oriented expert system 18 pages

RR-92-29

Zhaohur Wu, Ansgar Bernardi, Christoph Klauck:

Skeletel Plans Reuse: A Restricted Conceptual

Graph Classification Approach

13 pages

\section{DFKI Technical Memos}

TM-91-11

Peter Wazinski: Generating Spatial Descriptions for Cross-modal References

21 pages

TM-91-12

Klaus Becker, Christoph Klauck, Johannes Schwagereit: FEAT-PATR: Eine Erweiterung des D-PATR zur Feature-Erkennung in CAD/CAM 33 Seiten

TM-91-13

Knut Hinkelmann:

Forward Logic Evaluation: Developing a Compiler from a Partially Evaluated Meta Interpreter

16 pages

\section{TM-91-14}

Rainer Bleisinger, Rainer Hoch, Andreas Dengel:

ODA-based modeling for document analysis

14 pages

\section{TM-91-15}

Stefan Bussmann: Prototypical Concept Formation An Alternative Approach to Knowledge

Representation

28 pages

TM-92-01

Lijuan Zhang:

Entwurf und Implementierung eines Compilers zur Transformation von Werkstückrepräsentationen 34 Seiten

TM-92-02

Achim Schupeta: Organizing Communication and Introspection in a Multi-Agent Blocksworld 32 pages

TM-92-03

Mona Singh

A Cognitiv Analysis of Event Structure

21 pages

TM-92-04

Jürgen Müller, Jörg Müller, Markus Pischel,

Ralf Scheidhauer:

On the Representation of Temporal Knowledge

61 pages

TM-92-05

Franz Schmalhofer, Christoph Globig, Jörg Thoben The refitting of plans by a human expert

10 pages

TM-92-06

Otto Kühn, Franz Schmalhofer: Hierarchical

skeletal plan refinement: Task- and inference structures

14 pages 
An Interval-based Temporal Logic in a Multivalued Setting

Mathias Bauer

DFKI-RR-92-10 
This work has been supported by a grant from The Federal Ministry for Research and Technology (FKZ ITW-9000 8).

This work may not be copied or reproduced in whole or in part for any commercial purpose. Permission to copy in whole or in part without payment of fee is granted for nonprofit educational and research purposes provided that all such whole or partial copies include the following: a notice that such copying is by permission of Deutsches Forschungszentrum für Künstliche Intelligenz, Kaiserslautern, Federal Republic of Germany; an acknowledgement of the authors and individual contributors to the work; all applicable portions of this copyright notice. Copying, reproducing, or republishing for any other purpose shall require a licence with payment of fee to Deutsches Forschungszentrum für Künstliche Intelligenz. 

